\title{
Parallel electron-beam-induced deposition using a multi-beam scanning electron microscope
}

\author{
P. C. Post, A. Mohammadi-Gheidari, C. W. Hagen, ${ }^{\text {a) }}$ and P. Kruit \\ Delft University of Technology, Faculty of Applied Sciences, Lorentzweg 1, 2628CJ Delft, The Netherlands
}

(Received 24 June 2011; accepted 2 October 2011; published 26 October 2011)

\begin{abstract}
Lithography techniques based on electron-beam-induced processes are inherently slow compared to light lithography techniques. The authors demonstrate here that the throughput can be enhanced by a factor of 196 by using a scanning electron microscope equipped with a multibeam electron source. Using electron-beam induced deposition with $\mathrm{MeCpPtMe}_{3}$ as a precursor gas, $14 \times 14$ arrays of Pt-containing dots were deposited on a W/Si ${ }_{3} \mathrm{~N}_{4} / \mathrm{W}$ membrane, with each array of 196 dots deposited in a single exposure. The authors demonstrate that by shifting the array of beams over distances of several times the beam pitch, one can deposit rows of closely spaced dots that, although originating from different beams within the array, are positioned within $5 \mathrm{~nm}$ of a straight line. (C) 2011 American Vacuum Society. [DOI: 10.1116/1.3656027]
\end{abstract}

\section{INTRODUCTION}

Using resist-based electron beam lithography (EBL), one can routinely fabricate patterns down to $10 \mathrm{~nm}$, and even down to about $5 \mathrm{~nm}$ (Ref. 1) when using ultrathin resists and dedicated development processes. Using electron-beam-induced deposition (EBID), even smaller patterns of $1 \mathrm{~nm}$ in size can be written. ${ }^{2}$ In EBID, a focused electron beam dissociates precursor molecules adsorbed on a substrate surface, leaving a solid deposit on the surface and gaseous fragments that can be pumped away. The reader interested in EBID is referred to some recently published review papers. ${ }^{3-6}$ The advantage of EBID over EBL is that it is a direct deposition technique and provides a smaller minimum feature size. However, both EBL and EBID are inherently slow lithography techniques compared to light lithography techniques, because they are serial writing processes rather than parallel. However, in order to enhance the throughput, one could write with many electron beams in parallel. Several authors have proposed and/or built such multibeam lithography systems, ${ }^{7-20}$ which can be divided roughly into four types: (i) multiple optical columns with multiple sources, ${ }^{10-12}$ (ii) single column with multiple sources, ${ }^{13-15}$ (iii) single column with single source, ${ }^{16-22}$ and (iv) multiple cold field emitters in close proximity to the wafer. ${ }^{23}$ Although the latter system seems attractive, because it does not require any optics, it has not been demonstrated yet. The system we designed is a single column, single source system. We developed a multibeam electron source based on a standard single Schottky electron emitter mounted on a regular scanning electron microscope (SEM). This multibeam SEM (MBSEM) system distinguishes itself from other systems in that it projects an array of $14 \times 14$ focused beams onto a sample with a probe size and current per beam comparable to that of a standard single beam SEM, ${ }^{24,25}$ i.e., 196 beams, each with a $1 \mathrm{~nm}$ probe size and $30 \mathrm{pA}$ of current.

The objective of this article is to report the parallel direct deposition of dots using multibeam EBID. These experiments serve as a first test of the MBSEM as a multibeam EBID system.

a)Electronic mail: c.w.hagen@tudelft.nl

\section{EXPERIMENT}

\section{A. Multibeam SEM}

Although the design of the multibeam electron source and its integration in a standard SEM have been described in detail elsewhere, ${ }^{24-26}$ it is useful here to give a brief description of the MBSEM. In Fig. 1 a schematic overview of the system is shown. It consists of an FEI Nova-Nano 200 SEM equipped with the multibeam electron source module. The module is composed of two crucial components: (i) the multibeam source (MBS) and (ii) the accelerator lens (ACC). In the $\mathrm{MBS}$, the full emission cone of a single high brightness Schottky thermal field emission source is split up into an array of $14 \times 14$ focused microbeams by an aperture lens array (ALA). The combination of the ALA, which consists of a microfabricated Si membrane with apertures of $18 \mu \mathrm{m}$ diameter at a $25 \mu \mathrm{m}$ pitch, and the two macroelectrodes E-1 and E2 , is uniquely designed to correct for field curvature, to have low spherical aberration, and to minimize the chromatic deflection error. The ACC accelerates the beams to the required final energy and directs the beams to the SEM column. Although the system is designed for a variable magnification, for the present experiments we used a fixed magnification, with the first condenser lens (C2) switched off. The crossover at the current limiting variable aperture is imaged by the intermediate lens onto the coma-free plane of the ultrahigh resolution objective lens (UHR). Further demagnification of the probes is done by the UHR. The typical optical design parameters of this mode at a $15 \mathrm{keV}$ acceleration voltage are as follows: each beam has a $1 \mathrm{~nm}$ spot size at the sample, at a pitch of $360 \mathrm{~nm}$ at the sample, and a current of $31.4 \mathrm{pA}$ per beam, and the total footprint of the $14 \times 14$ array is $(5 \times 5) \mu \mathrm{m}^{2}$.

\section{B. Multibeam EBID}

Focusing the array of 196 beams onto a sample is not straightforward. Using the regular Everhart-Thornley secondary electron detector, one collects the secondary electrons of all 196 beams simultaneously, such that one beam cannot be distinguished from the others. Therefore, we used a transmission detector in combination with a membrane 


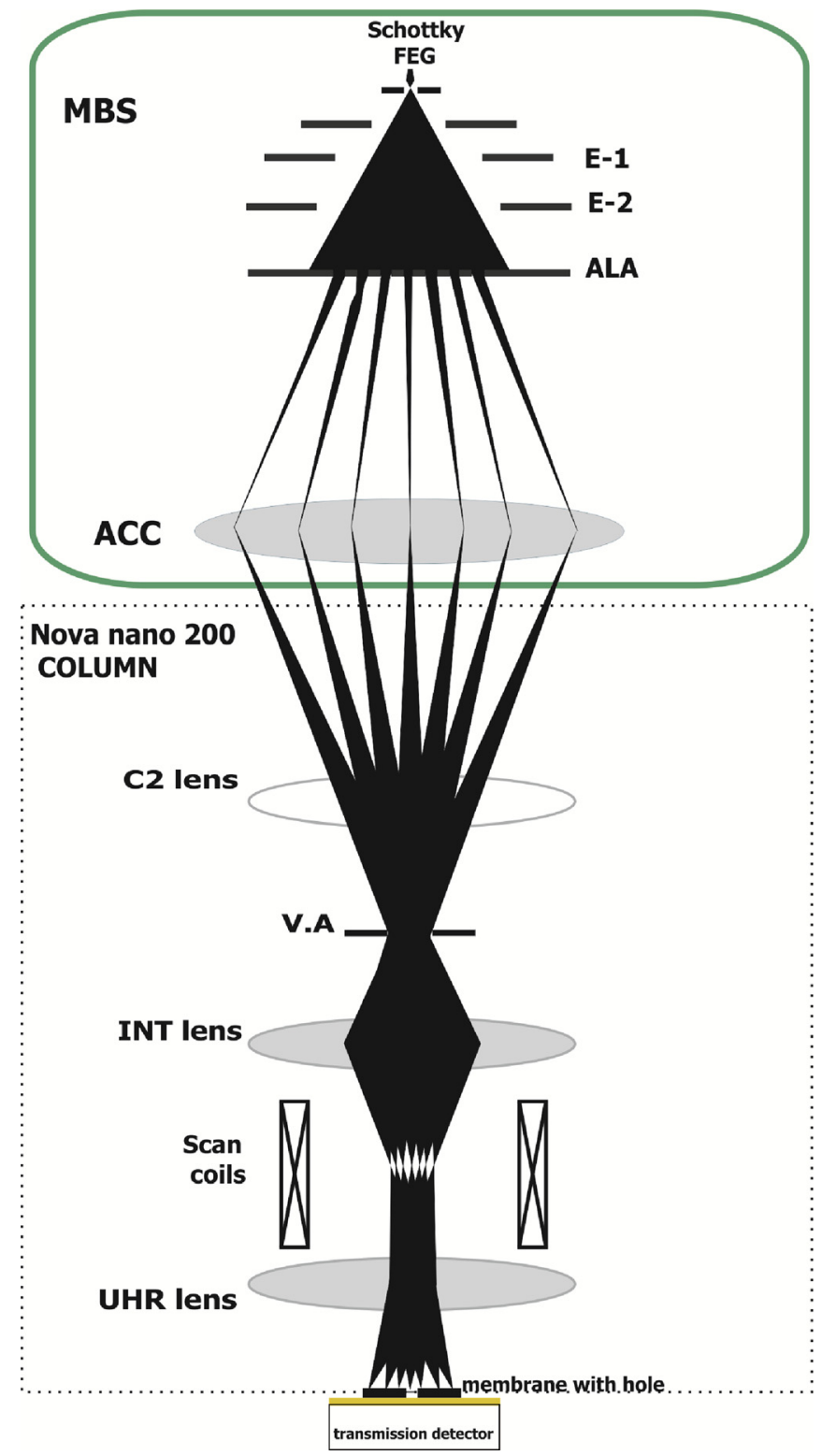

FIG. 1. (Color online) Schematic drawing of the multibeam SEM mode when the $\mathrm{C} 2$ lens is switched off. This is the mode in which the deposition experiments were performed. The components indicated are the macroelectrodes E-1 and E-2, the aperture lens array (ALA), the accelerator lens (ACC), the condenser lens (C2), the variable aperture (VA), the intermediate lens (INT), and the ultrahigh-resolution objective lens (UHR). The beams are focused using the transmission detector below the membrane sample with apertures.

sample with an aperture. The membrane is a $\mathrm{W} / \mathrm{Si}_{3} \mathrm{~N}_{4} / \mathrm{W}$ sandwich, with layer thicknesses of $200 \mathrm{~nm} / 50 \mathrm{~nm} / 200 \mathrm{~nm}$, sufficiently thick to stop $15 \mathrm{keV}$ electrons. The aperture is just a hole in the membrane and is created by focused ion beam milling, using an FEI quanta 3D FEG dual beam system. A gallium ion beam was used at an energy of $30 \mathrm{keV}$ and a beam current of $30 \mathrm{pA}$. The milling process was observed using the electron beam and secondary electron detection, and the end point of the process was determined by measuring the transmission signal of the transmission detector below the membrane. Several holes were milled in the membrane at least $20 \mu \mathrm{m}$ apart, i.e., at a distance larger than the size of the array of beams. In order to keep multiple beams from passing through the aperture, the aperture diameter (typically about $250 \mathrm{~nm}$ ) has to be smaller than the beam pitch of $360 \mathrm{~nm}$.

Scanning the array of 196 beams over a single aperture and detecting the bright field transmission signal results in an image of 196 bright spots. The best focusing of the beams was obtained by optimizing the edge contrast of these spots.

For the deposition, we used the platinum precursor methyl-cyclopentadienyl-trimethyl-platinum $\left(\mathrm{MeCpPtMe}_{3}\right.$, CAS: 94442-22-5) as a precursor gas. The specimen chamber was filled with precursor gas at a pressure of $2.5 \times 10^{-5}$ mbar. After the beams were focused, the membrane was displaced (typically by $10 \mu \mathrm{m}$ ) in order to obtain a fresh deposition area away from the aperture. The defocus due to the displacement was checked by displacing the membrane to a different aperture, even as far as $100 \mu \mathrm{m}$ away, and no severe defocus was observed. To expose the adsorbed precursor layer, we used Labview-based homebuilt patterning software that controls the position of the array of beams and the exposure time. As the microscope does not have a fast blanker, the beam was unblanked just before the start of the writing sequence and then moved to the deposition area where the writing sequence was completed, after which the beam was moved away from the deposition area, where the beam was blanked. In this way, spurious deposition close to the deposition area during the relatively slow blanking time is avoided. After the deposition, the gas was pumped out and the dot arrays were imaged by taking single-beam SEM images in our FEI quanta 3D FEG Dual Beam system.

\section{RESULTS AND DISCUSSION}

Figure 2 shows a single beam image of an array of Pt-containing EBID dots, deposited with a single exposure in the MBSEM. The exposure time was $20 \mathrm{~s}$. This is a relatively long exposure time, resulting in rather large deposits. This is done on purpose because it leads to better contrast images than when tiny sub-10 nm dots are deposited, and it makes it easier to judge the quality of the array of beams. The dots have a diameter of about $70 \mathrm{~nm}$, and the average pitch is $436 \mathrm{~nm}$. The total field of $14 \times 14$ dots measures $(5.7 \times 5.7)$ $\mu \mathrm{m}^{2}$. On the left-hand side of the image, the dots still have a slightly cometlike shape. This is because the crossover of the beams is not imaged exactly in the coma-free plane of the objective lens. Also, the fact that some of the beams are missing is due to a slight misalignment of the optical system.

To demonstrate the possibilities of multibeam EBID, we did another experiment in which we shifted the array of beams five times over a relatively large distance of about $2 \mu \mathrm{m}$ (4.6 times the beam pitch), each time exposing the sample for $15 \mathrm{~s}$. The single beam SEM image of the resulting assembly of dots is shown in Fig. 3. The markers in Fig. 3 serve as an aid to help the observer easily determine which dot originates from which exposure. The square markers indicate dots deposited by the same beam at five successive positions of the array of beams, and the hexagonal-, star-, and circle-shaped markers indicate three neighboring dots, 


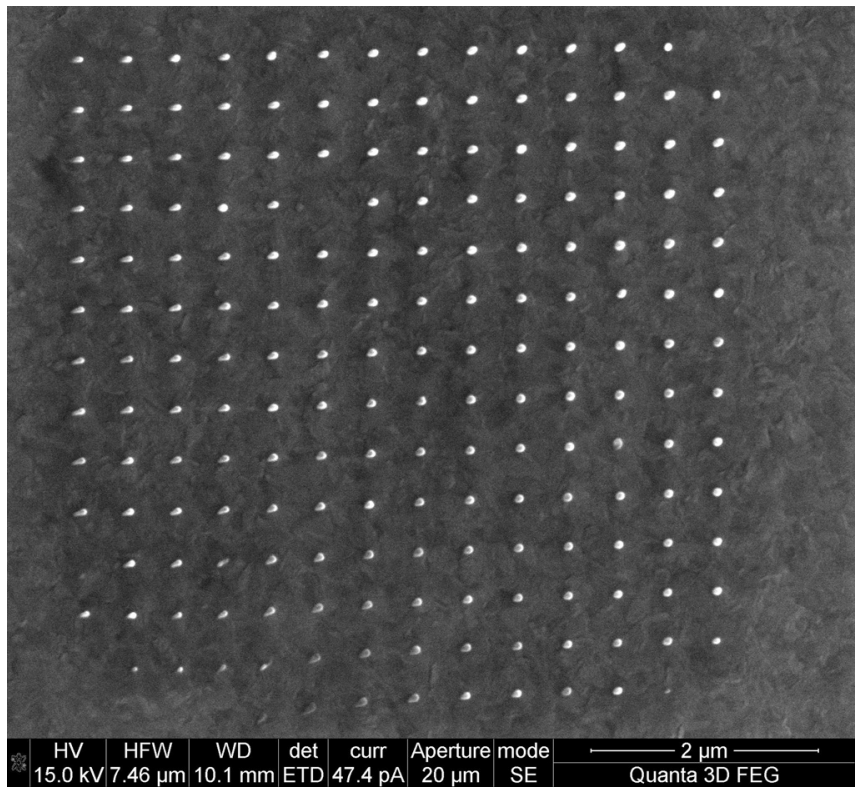

FIG. 2. Single-beam SEM image of an array of EBID dots grown on the W/ $\mathrm{Si}_{3} \mathrm{~N}_{4} / \mathrm{W}$ membrane sample using a single $20 \mathrm{~s}$ exposure in the multibeam $\mathrm{SEM}$ at $15 \mathrm{keV}$. The precursor gas used was $\mathrm{MeCpPtMe}_{3}$, at a pressure of $2.5 \times 10^{-5}$ mbar. A few dots are missing, and the dots on the left-hand side reveal the presence of coma. This is due to misalignment and the fact that the crossover of the beams is not imaged exactly in the coma-free plane of the objective lens.

deposited at the five different positions of the array of beams. It is easily seen from Fig. 3 that rows of dots can be created in which adjacent dots do not originate from adjacent beams in the array of beams (see the short line connecting three dots in a row). When choosing the proper direction in which the array of beams is to be shifted, dots exposed by beams far apart in the array can be grouped together to form regular rows of dots over large distances, or even continuous lines if the dots overlap. The uniformity of the resulting pattern depends on the accuracy of the array positioning and on the regularity of the array of beams, i.e., on the pitch uni-

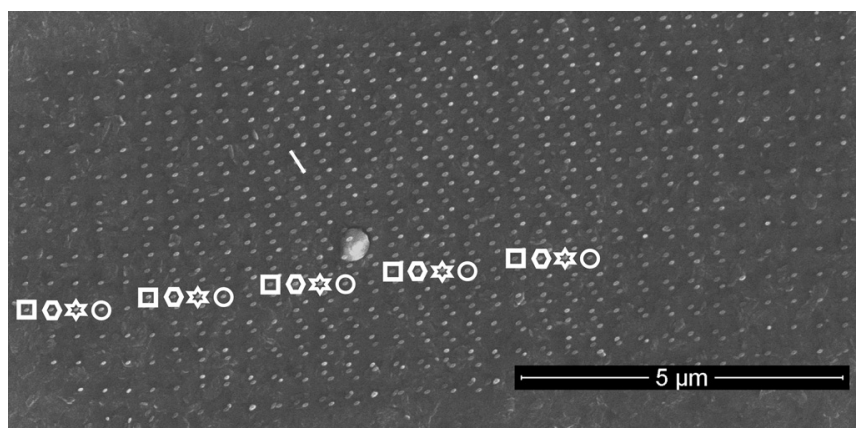

FIG. 3. Single-beam SEM image of multibeam EBID dots grown in five successive $15 \mathrm{~s}$ exposures in the multibeam SEM at $15 \mathrm{keV}$, with each exposed position shifted by about $2 \mu \mathrm{m}$. The square markers indicate a particular dot written by the same beam in five successive exposures, and the hexagons, stars, and circles indicate three neighboring dots. The potential formation of rows of dots is illustrated by the small line connecting three lined-up dots written by three different beams. The precursor gas used was $\mathrm{MeCpPtMe}_{3}$, at a pressure of $2.5 \times 10^{-5}$ mbar. formity. When attempting to determine the orientation of the array such that the displacement of the array of beams can be chosen along the preferred direction, in situ imaging with the MBSEM is very helpful. Of course, there is only one secondary electron detector, which collects the signals of all 196 beams simultaneously. The resulting image is a convolution of the deposited dot array and the $14 \times 14$ array of beams that is scanned over the dot array. Because both the deposition and the imaging are done with the same array of 196 beams, the resulting image still contains useful information, such as the orientation of the array of dots, provided the pitch variation within the array of beams is sufficiently small. In Fig. 4 we demonstrate the deposition of a linear row of $150 \mathrm{~nm}$ spaced dots by shifting the array five times over a distance of $0.84 \mu \mathrm{m}$ (twice the beam pitch) in the direction indicated by the circles in Fig. 4. The line drawn along the ten dots serves as a guide for the eye in order to demonstrate that the ten dots, all originating from beams located far apart within the array of beams, can be deposited within a distance of about $5 \mathrm{~nm}$ from a straight line, as is easily judged from the width of the line, which is $20 \mathrm{~nm}$.

We demonstrated the potential of multibeam EBID as a direct lithography technique, with a 196-fold speed enhancement with respect to single beam EBID. The meaning of this becomes clear when considering large area deposition. As an example, suppose we were to upscale the size of the array of dots in Fig. 3 to an area of $(100 \times 100) \mu \mathrm{m}^{2}(\sim 140000$ dots $)$. In a single beam EBID process, this would take 24 days, whereas with multibeam EBID this can be done in only $3 \mathrm{~h}$ !

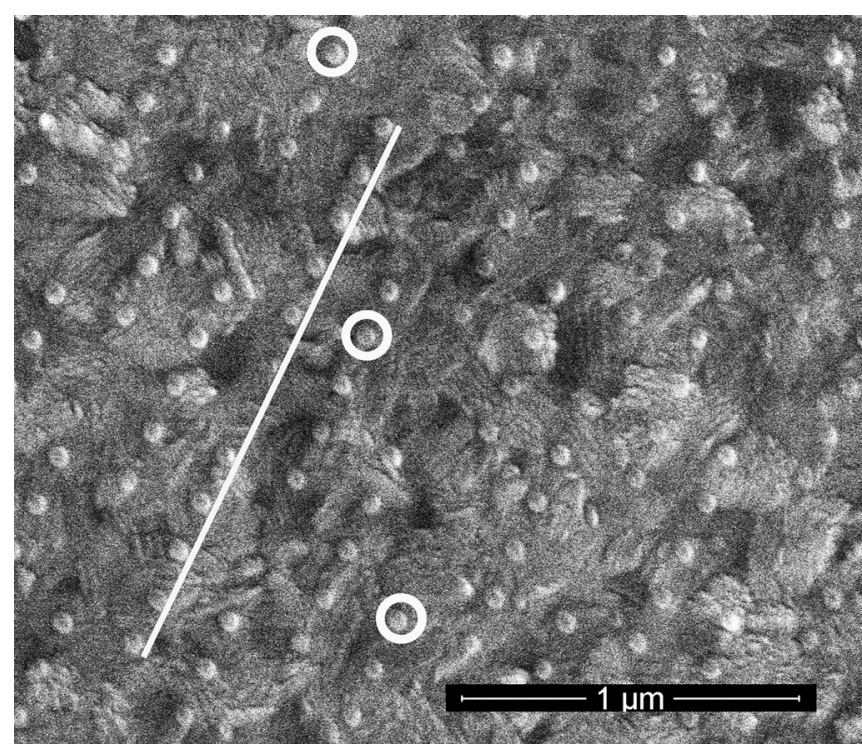

FIG. 4. Single-beam SEM image of five successive $15 \mathrm{~s}$ exposures in the multibeam SEM at $15 \mathrm{keV}$, shifted with respect to each other by $0.84 \mu \mathrm{m}$ in the direction of the imaginary line connecting the three circles. The precursor gas used was $\mathrm{MeCpPtMe}_{3}$, at a pressure of $2.5 \times 10^{-5}$ mbar. The $20 \mathrm{~nm}$ thick line is a guide for the eye, to help the viewer judge how well the dots are positioned with respect to a mutually connecting straight line (in this case it is better than $5 \mathrm{~nm}$ ). As each dot within an array of five dots originates from a different beam, this demonstrates the patterning capability of the multibeam SEM. 


\section{SUMMARY AND CONCLUSIONS}

We used a scanning electron microscope equipped with a multibeam electron source for the electron-beam-induced deposition of 196 dots simultaneously. We have learned how to operate the instrument in order to do parallel EBID, which introduces some new difficulties in terms of focusing and imaging. We have demonstrated that by shifting the array of beams over distances of several times the beam pitch, we can deposit rows of closely spaced dots in which dots originating from different beams within the array are positioned within $5 \mathrm{~nm}$ of a straight line. This shows that the variation in pitch within the array of beams is smaller than $5 \mathrm{~nm}$ and that we have good control over the positioning of the array of beams. The dots we deposited were not the smallest possible ones, for reasons of visibility in the images. It was more important here to demonstrate the potential of multibeam EBID, in terms of patterning capability and speed enhancement, than to obtain sub- $10 \mathrm{~nm}$ resolution. In order to develop the multibeam SEM into a full lithography tool, we plan to put a microfabricated deflector plate at the position of the accelerator lens to obtain beam blanking of each beam individually. Furthermore, the controls of the new multibeam source optics have to be integrated in the control electronics of the SEM, in order to facilitate easy operation of the microscope and enable rapid switching between multibeam mode and single-beam mode. The latter is really desirable for imaging purposes.

\section{ACKNOWLEDGMENTS}

We gratefully acknowledge V.G. Kutchoukov for fabrication of the sandwich samples, F. Berwald for developing the patterning software, and the support provided by NanoNed and the EU Cost Action CM0601 "Electron Controlled Chemical Lithography" (ECCL).
${ }^{1}$ J. K. W. Yang, B. Cord, H. Duan, K. K. Berggren, J. Klingfus, S. W. Nam, K. B. Kim, and M. J. Rooks, J. Vac. Sci. Technol. B 27, 2622 (2009).

${ }^{2}$ W. F. van Dorp, B. van Someren, C. W. Hagen, P. Kruit, and P. A. Crozier, Nano Lett. 5, 1303 (2005).

${ }^{3}$ N. Silvis-Cividjian and C. W. Hagen, Advances in Imaging and Electron Physics (Elsevier, Amsterdam, 2006), Vol. 43.

${ }^{4}$ K. Furuya, Sci. Technol. Adv. Mater. 9, 014110 (2008).

${ }^{5}$ I. Utke, P. Hoffman, and J. Melngailis, J. Vac. Sci. Technol. B 26, 1197 (2008).

${ }^{6}$ W. F. van Dorp and C. W. Hagen, J. Appl. Phys. 104, 081301 (2008).

${ }^{7}$ B. Roelofs and J. Barth, Microelectron. Eng. 2, 259 (1984).

${ }^{8}$ M. McCord, J. Vac. Sci. Technol. B 15, 2125 (1997).

${ }^{9}$ T. Chang, M. Mankos, K. Lee, and L. Muray, Microelectron. Eng. 57-58, 117 (2001)

${ }^{10}$ L. P. Muray, J. P. Spallas, C. Stebler, K. Lee, M. Mankos, Y. Hsu, M. Gmur, and T. H. P. Chang, J. Vac. Sci. Technol. B 18, 3099 (2000).

${ }^{11}$ T. R. Groves and R. A. Kendall, J. Vac. Sci. Technol. B 16, 3168 (1998).

${ }^{12}$ T. Haraguchi, T. Sakazaki, T. Satoh, M. Nakano, S. Hamaguchi, T. Kiuchi, H. Yabara, and H. Yasuda, J. Vac. Sci. Technol. B 22, 985 (2004).

${ }^{13}$ E. Yin, A. D. Brodie, F. C. Tsai, G. X. Guo, and N. W. Parker, J. Vac. Sci. Technol. B 18, 3126 (2000).

${ }^{14}$ S. Tanimoto, Y. Someda, M. Okumura, H. Ohta, Y. Sohda, and N. Saitou, Jpn. J. Appl. Phys., Part 142, 6672 (2003).

${ }^{15}$ S. T. Coyle et al., J. Vac. Sci. Technol. B 22, 501 (2004).

${ }^{16}$ H. Yasuda, S. Arai, J.-I. Kai, Y. Ooae, T. Abe, S. Maruyama, and T. Kiuchi, J. Vac. Sci. Technol. B 14, 3813 (1996).

${ }^{17}$ G. Winograd, V. Krishnamurthi, R. Garcia, L. H. Veneklasen, M. Mankos, and F. Pease, J. Vac. Sci. Technol. B 18, 3052 (2000).

${ }^{18}$ M. Muraki and S. Gotoh, J. Vac. Sci. Technol. B 18, 3061 (2000).

${ }^{19}$ N. Nakasuji, S. Yoshikawa, T. Satake, and N. Noji, Jpn. J. Appl. Phys., Part 1 44, 5570 (2005).

${ }^{20}$ O. Kamimura et al., J. Vac. Sci. Technol. B 25, 140 (2007).

${ }^{21}$ J. Basu, C. B. Carter, R. Divakar, V. B. Shenoy, and N. Ravishankar, Appl. Phys. Lett. 93, 133104 (2008).

${ }^{22}$ H. M. Kim, M. H. Lee, H. S. Lee, J. S. Wi, K. Lim, and K. B. Kim, J. Vac. Sci. Technol. B 27, 2553 (2009).

${ }^{23}$ L. Dong, F. Arai, and T. Fukuda, Appl. Phys. Lett. 81, 1919 (2002).

${ }^{24}$ A. Mohammadi-Gheidari, C. W. Hagen, and P. Kruit, J. Vac. Sci. Technol. B 28, C6G5 (2010).

${ }^{25}$ A. Mohammadi-Gheidari and P. Kruit, Nucl. Instrum. Methods Phys. Res. A 645, 60 (2011).

${ }^{26}$ Y. Zhang and P. Kruit, Phys. Procedia 1, 553 (2008). 